\title{
Jahreskongress der DGH 2013 - Wo steht die Handchirurgie im Zeitalter der Evidenz basierten Medizin?
}

\section{Report from the Annual Meeting 2013 in Düsseldorf}

Der Jahreskongress beschäftigte sich gleich zu Beginn mit der Frage nach der wissenschaftlichen Basis der alltäglichen handchirurgischen Praxis.

Herr Antes führte dabei, - wie unten aufgeführt -, in seinem exzellenten Übersichtsvortrag durch die Irrungen und Fallen der Evidence based Medicine (EVBM):

\section{Was ist eigentlich EVBM? \\ $\nabla$}

Hierunter versteht man den [....] vernünftigen Gebrauch der gegenwertig bestverfügbaren externen wissenschaftlichen Evidenz für Entscheidungen der medizinischen Versorgung individueller Patienten (Sackett et al.).

Und hier beginnt das Problem, denn der eigentliche Goldstandard der EVBM, indem zeitgleich zur Therapie auch eine Alternativ,- bzw. Kontrollgruppe hinsichtlich des Outcomevergleiches generiert wird, gelingt aus ethischen Gründen oder aufgrund der Fallzahlgrößen in der Handchirurgie oftmals nicht, zumal zum Ausschluss von sogenannten Mischeffekten auch nur ähnliche Patienten eingeschlossen werden sollten.

Auch kann eine Studie mit hohem evidenz based level aufgrund von systemischen Fehlern schlechter sein als z. B. eine gute Fallserie z.B. wenn Outcomeparameter gewählt werden, die gar nicht zur Fragestellung passen.

Ein weiteres Problem stellt der Transfer der Forschungsergebnisse in die klinische Praxis dar. Bei jährlich 600000 Studien und davon 20000 randomisierte, kontrollierte Studien erreichen 50\% der Forschungsergebnisse wegen nicht erfolgter Publikationen die klinischen Anwender nicht. Das selektive Publizieren führt in der Konsequenz zu einer positiven Überschätzung der beschriebenen Therapien um $20-40 \%$.

Viele neue Ansätze sollen diese Lücke nun schließen und die Forscher verpflichten auch ihre Ergebnisse zu publizieren. Dies reicht von der Registrierung zum Zeitpunkt der Ethikkommission bis zu gesetzlichen Vorgaben in den U.S.A.

\section{Was bleibt als Fazit?}

Das Studiendesign (Methodik) bestimmt ebenso wie der EVB-Level über die klinische Relevanz und nur durch Publikationen der Ergebnisse kann der konsequente Transfer in den klinischen Alltag gelingen.

\section{Was aber bedeutet dies für die Handchirurgie?}

Auch auf diesem Kongress hat sich gezeigt, dass trotz großem Aufwand einer der größten Probleme die jeweils geringe Fallzahl der Untersuchungsgruppen und der EVB Level darstellten. Ein Großteil der Beiträge beschrieb Fallserien oder Methodikanalysen. Von 41 Beiträgen waren 7 Übersichtsreferate, 12 Fallserien, 4 prospektive, 7 retrospektive Studien, 2 Fallvorstellungen und 9 Methodikanalysen zu differenzieren.

Die mittlere Patientenanzahl der Fallserien betrug knapp 19 Patienten, bei den prospektiven Studien 37 und bei den retrospektiven Studien im Schnitt 38 Patienten (Anm.: Ausnahme Ulnokarpale Bandverletzungen). Bedenkt man nun noch, dass die Daten häufig über einen Zeitraum von mehreren Jahren und die Auswertung mit Untergruppierungen erfolgte, so minimiert sich folgerichtig die akute klinische Relevanz der gewonnenen Ergebnisse.

Den einzigen Weg aus diesem Dilemma bildet der Zusammenschluss von Forschungsgruppen mit gleichen Interessen. Dies behebt auch den Missstand der repetitiven Studien gleichen Inhaltes, die über die Jahre immer wieder neu aufgelegt werden. Damit eine solche Vernetzung möglich ist wird Anfang 2014 eine entsprechende Plattform auf der Homepage der DGH eingerichtet werden, auf der alle Forschungsgruppen die Möglichkeit erhalten ihr Projekt selber vorzustellen und ggf. für Kooperationspartner oder Doktoranden zu werben. So können in gegenseitigem Miteinander auch die jeweiligen Ressourcen der anderen Kliniken optimal genutzt werden.

Wenn sich dieser Ansatz zur Kooperation durchsetzen kann, wird es sicherlich bald möglich sein qualitativ hochwertige Studien mit hoher Fallzahl und klinischer Relevanz zu generieren.

\section{Was gab es sonst noch Neues auf der DGH? \\ $\nabla$}

Der Kongress beschäftigte sich in diesem Jahr neben der EVBM mit dem Thema der Frakturbehandlung, den lokalen Lappenplastiken, ligamentären Verletzungen und freien Themen.

In der Sitzung der EVBM wurde zunächst am Beispiel von Radialisparesen versucht zu belegen, dass die EVBM nicht alle individuellen Faktoren berücksichtigt, die das therapeutische Vorgehen beeinflussen würden, sodass die individuelle Entscheidung höher zu stellen sei als Algorithmen oder EVBM. Da die Definition der EVBM aber einen „vernünftigen, Gebrauch des Wissens für „individuelle,, Patienten propagiert liegt kein Widerspruch in der Anwendung der EVBM und individuellen Entscheidungen unter Berücksichtigung der EVBM. Interessant war auch die Untersuchung von 48 Hand- und Handgelenkscores auf ihre Inhalts-, Konstruktions-, und Kriteriumsvalidität. Der Autor stellte fest, dass nur 7 der 48 Scores alle Validitätskriterien erfüllten, sodass bei Studienplanungen eine größere Sorgfalt auf die Auswahl der Outcomescores gelegt werden müsse. In dem Abschnitt der Frakturbehandlung wurde neben perkutanen und intramedullären Verfahren zum einen eine neue Rückzugstechnik zur Versorgung von dorsalen Endgliedbasisfrakturen und eine experimentelle Studie zur elektromagnetisch navigierten Schraubenosteosynthese bei Skaphoidfrakturen (=ENS) vorgestellt. Bei den Endgliedbasisfrakturen wurde eine PDS Cerclage zunächst mit der Nadel durch den Knochen und dann durch den Fragmentsehnenanteil gefädelt und das Fragment so reponiert. Es wurden $53 \mathrm{~Pa}-$ tienten mit „gutem Erfolg“ bei sehr unterschiedlichen Indikationen behandelt, die Bewegungsausmasse nach Versorgung jedoch nicht beschrieben. An Komplikationen wurden ein Fadenriß und ein schleichender Infekt benannt.

Bei dem Vortrag zur elektromagnetisch navigierten Schraubenplatzierung bei Skaphoidfrakturen wurden 6 Kadaverhände konventionell mit dem Bildwandler und 6 mit der neuen Technik (ENS) versorgt. Dabei wurde neben der OP Zeit auch die Lage der Schraube im CT verifiziert und mit der Idealachse verglichen. 


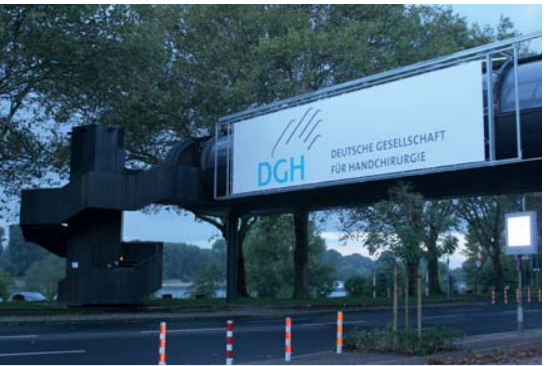

Abb. 1 Der Kongress fand dieses Jahr direkt am Rhein statt. (Abbildung mit freundlicher Genehmigung der Intercongress $\mathrm{GmbH}$ ).

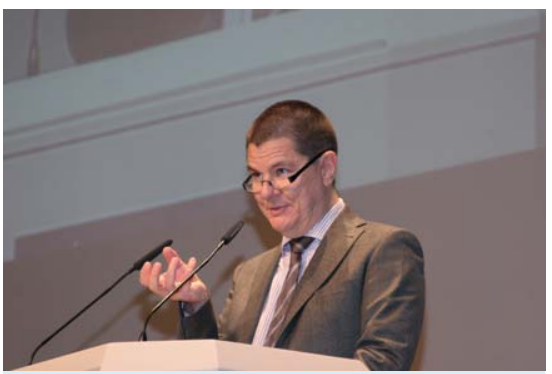

Abb. 2 Kongresspräsident Joachim Windolf sorgte für ein buntes Programm. (Abbildung mit freundlicher Genehmigung der Intercongress $\mathrm{GmbH}$ ).

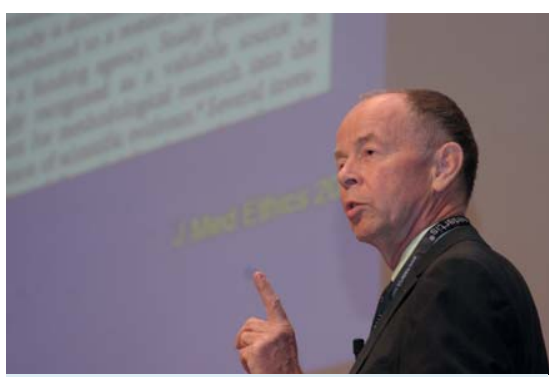

Abb. 3 Herr Antes führte gekonnt durch die Welt der EVB. (Abbildung mit freundlicher Genehmigung der Intercongress $\mathrm{GmbH}$ ).

Eine Empfängersonde wurde unter Bildwandlerkontrolle am distalen Pol über eine Inzision platziert und dann von antegrad über den dorsalen Zugang die Schraube navigiert eingebracht. Mit der ENS betrug die OP Zeit im Schnitt 7 min weniger, die Idealachse wurde präziser getroffen und die Durchleuchtungszeit war geringer. Dennoch verbleibt derzeit noch das
Problem, dass nur nicht dislozierte Frakturen über einen dorsalen Zugang mit der ENS versorgt werden könnten.

Der Freitagvormittag stand unter dem Thema der lokalen Lappenplastiken. Nach einem informativen Übersichtsvortrag von Prof. G. Germann wurden verschiedene Fallserien zur Defektdeckung von palmaren Weichteildefekten mittels Insel,- und Crossfingerlappen vorgestellt und des Weiteren der seltene Fall eines Hidradenokarzinoms aufgearbeitet.

Es schloss sich die Sitzung der freien Vorträge an. Interessant war neben der Vorstellung des Designs einer Pilotstudie zur Optimierung der Stammnervenrekonstruktion durch Elektrostimulation mit implantierten Elektroden, eine Studie, die die Regenerationsergebnisse sensibler Digitalnerven nach Naht, Nerventransplantation und Interposition von Venenmuskelinterponaten untersuchte. Diese 3 Gruppen wurden nach 6 Monaten verglichen und es fanden sich im Vergleich zwischen Venenmuskelinterponaten und Nerveninterponaten keine Unterschiede (altersunabhängig) in der erreichten 2 Punktediskrimination $(5,0-5,5 \mathrm{~mm})$, jedoch verbleibende Sensibilitätsdefizite an der Hebestelle bei Transplantation. Die Fallzahl ist jedoch auch hier noch zu gering und die Mischeffekte sind zu groß, um schon eine definitive Aussage für die Klinik tätigen zu können. Ein weiterer interessanter Vortrag untersuchte die potentielle Schädigung von Knorpelgewebe bei der Arthroskopie im Rahmen der Radiofrequenztherapie. Geht man von einer Gewebeschädigung bei $50^{\circ} \mathrm{C}$ aus, so erreichte die Messung bei monopolarem Einsatz $50^{\circ}$ und bei Bipolaren bis zu $70^{\circ}$. Diese Temperaturen werden nach 10 s erreicht, sodass als klinisches Fazit eine hohe Spülrate und möglichst kurze monopolare statt bipolare Anwendungen empfohlen wurden.

Die DGH endete am Samstag mit den freien Themen und ligamentären Bandverletzungen. In einer großen retrospektiven Studie wurde untersucht inwieweit sich Läsionen des ulnokarpalen Bandkomple- xes vor einer Arthroskopie (ASK) vorhersagen lassen. Dabei wurden die Ergebnisse der präoperativen klinischen und auswärtigen radiologischen (MRT) Untersuchung mit dem tatsächlichen ASK Befund korreliert und der positiv prädiktive Wert ermittelt. Letztlich lag dieser zwischen 0,55 und 0,59 , sodass diese Verfahren in diesem Setting nicht dazu geeignet waren eine Läsion sicher vorherzusagen oder auch auszuschließen.

Interessant war auch eine Studie zur arthroskopischen Synovialektomie der Fingergrund (=MP),- und Mittelgelenke (=PIP) bei Arthrose und rheumatischer Arthritis (=RA). Während diese Methode bei der RA zu einer guten Zufriedenheit und Schmerzreduktion führte (MP>PIP), konnte bei der Arthrose nur bei geringerer Ausprägung und bei den MP Gelenken eine Verbesserung erzielt werden.

Insgesamt hat auch dieser Jahreskongress 2013 wieder viele neue und interessante Ansätze beinhaltet. Um eine patientenorientierte Forschung zu etablieren, die eine klinische Konsequenz für die Praxis implementiert wäre es wünschenswert, dass künftig die handchirurgisch Forschenden noch näher zusammenrücken und - arbeiten. Wir hoffen, dass ab 2014 die neue Plattform auf der DGH -Homepage hierfür eine Hilfe darstellt und die an ähnlichen Themen wissenschaftlich Interessierten zusammenführt.

\author{
Bibliografie \\ DOI http://dx.doi.org/ \\ 10.1055/s-0033-1361123 \\ Handchir Mikrochir Plast Chir \\ 2013; 45: 379-380 \\ (c) Georg Thieme Verlag KG \\ Stuttgart · New York \\ ISSN 0722-1819

\section{Korrespondenzadresse} \\ Dr.med. Caroline Dereskewitz \\ Abteilung für Handchirurgie, Plastische und \\ Mikrochirurgie Berufsgenossenschaftliches \\ Unfallkrankenhaus Hamburg Bergedorfer Straße 10 \\ 21033 Hamburg \\ c.dereskewitz@buk-hamburg.de
}

